\title{
Measurements of Body Movement in Chick Embryos During Early Stages
}

\author{
Kenji Moriya $^{a^{*}}$ and Yuya Chiba ${ }^{b}$ \\ a Department of Production Systems Engineering, National Institute of Technology, Hakodate College. \\ Tokura-cho 14-1, Hakodate City, Hokkaido, Japan 042-8501 \\ ${ }^{\mathrm{b}}$ Support Center for Engineering Education, National Institute of Technology, Hakodate College. \\ Tokura-cho 14-1, Hakodate City, Hokkaido, Japan 042-8501 \\ * Corresponding author: moriya @ hakodate-ct.ac.jp
}

\begin{abstract}
In this study, we investigate developmental patterns of body movements that may play roles in normal embryonic growth using direct recordings of chick embryos during the early stages of development (72-125 h of incubation). In three individuals, embryonic body movements occurred intermittently and irregularly after 72-h incubation, and frequencies and sizes of movements increased with embryonic growth. Additionally, patterns of the body movements became periodic at $85 \mathrm{~h}$ in all individuals, and cyclic periods of body movements were then shortened and periodicities of movements gradually disappeared with embryonic development. If cyclic body movements are a common requirement during normal chick embryo growth, continuous monitoring of body movements could be used to predict abnormal embryonic growth.

Future studies are required to investigate distinctive body movements and developmental patterns during embryonic growth in disease models and under various environmental conditions, such as under hypoxia.
\end{abstract}

Keywords: Early stages embryogenesis, Embryonic body movement, Template matching.

\section{Introduction}

\subsection{Chick embryo as models of fetal development}

Because chick embryos have all the nutrition necessary for growth inside their shells, the external conditions required for development are merely temperature and oxygen, leading to multiple advantages for studies of fetal development. Among these, embryonic physiological parameters that are not influenced by maternal health conditions can be measured, and assessments of responses to changes in temperature, humidity, and oxygen concentrations can be made, as shown previously ${ }^{(1-3)}$. Fertile chicken eggs can also be easily obtained from chicken farmers and physiological parameters in chick embryos have been considered suitable and independent indicators of embryonic development at all incubation periods, particularly in studies of cardiovascular system development ${ }^{(4-9)}$. Although measurements of bio-signals during the early stages of pregnancy in mammals are extremely difficult, those of early stages (until day-7) in chick embryos can be achieved with relative ease. Hence, as models of fetal development, chick embryos offer significant advantages.

\subsection{Investigation of body movement patterns in chick embryos during the early stages of development}

It is well established that heart tissues in chick embryos are formed after about $30 \mathrm{~h}$ incubation, and that other organs, such as chorioallantoic membranes, are under development at this early stage. However, no continuous and direct in ovo video recordings of chick embryos have been published previously. In a previous study, we developed a system for continuously recording chick embryo developments over long periods using a small Charge Coupled Device (CCD) camera mounted on the egg, and investigated physiological developments of chick embryos, including those of heart structures and blood vessels, with minimal or no sacrifice. Although cardiac signals are barely detectable using electrocardiograms during early stages of chick embryogenesis, we determined instantaneous heart rates during the early stages using video images that were directly recorded using our system ${ }^{(10-12)}$. 
A recent study indicated that mortality rates of human fetuses are greater among those with significantly reduced body movements ${ }^{(13)}$. Therefore, we developed a system for assessing embryonic body movements from continuous and direct recordings of chick embryos, and investigated developments of body movement patterns during the early stages of chick embryogenesis.

\section{Experimental methods}

\subsection{Direct recording of embryonic body movements}

A schematic and a photograph of the body movement recording system are presented in Fig. 1. Because the details of the direct recording system are described in a previous study ${ }^{(10-12)}$, we describe the system only briefly herein. To mimic general incubation conditions of eggs, incubation and recording procedures were performed in a constant temperature incubation at approximately $38{ }^{\circ} \mathrm{C}$ with $50 \%$ humidity. Holes of approximately $1.5 \mathrm{~cm}$ in diameter were made on the tops of egg shells (air-cell side), and a CCD camera (Type MTV-5366ND, Akiduki Denshi Tsusho Co. Inc., effective pixels; $352 \times 240$ ) was attached with a connecting plastic tube. Because defective seals between egg shells and the spacer for the CCD camera might cause significant physiological and/or biological adversities during embryonic development, sealing was performed very carefully. Additionally, adequate circulation of air was required to promote heat distribution to the CCD camera, and this was achieved using forced-draft incubation during experiments. Because previous studies report body movements of chick embryos starting from around $80 \mathrm{~h}$ of incubation, we started continuous monitoring experiments at $72 \mathrm{~h}$ (day-3) incubation. Video images were captured at 30 frames/s (i.e., with a sampling frequency of $30 \mathrm{~Hz}$ ) using
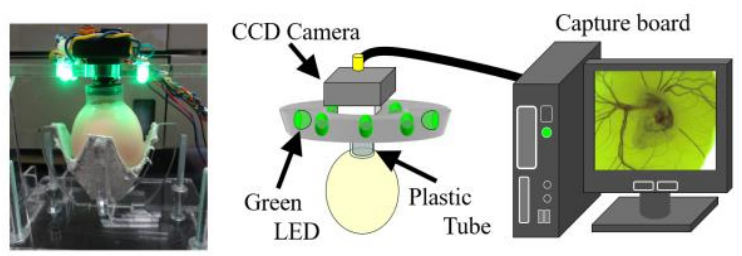

Fig. 1. The direct recording system for chick embryogenesis; the green LED was selected to emphasize the heart and blood vessels. The CCD camera was attached over a hole at the blank end of the egg (air-cell side) using a plastic tube, and was then glued to prevent air leaks and evaporation. Video images were recorded using a video capture a CCD camera with a video capture board attached to a computer via a coaxial cable. Images were saved for analyses in MPEG format. Although these procedures were invasive, previous experiments showed successful hatching at normal incubation times after closure of the hole in the top of the egg using a nylon-wrap to prevent evaporation (unpublish data).

\subsection{Quantitative determinations of body movements}

We developed a system for measuring sizes and frequencies of body movements using image processing tools. During the early stages of embryogenesis, whole embryos did not move, and embryonic movements were initially observed as those of the embryo's head. Thus, we calculated distances between embryo head positions as an indicator of body movements. Because body movement patterns develop with embryonic growth, those in the first 5 min of every $1 \mathrm{~h}$ of incubation were investigated. In the first 5 min of 1-h incubation time points, images were extracted after continuous video recording and were divided into 1-h segments. Locations of embryo heads were calculated using the template-matching method in the Open Source Computer Vision library (Open CV), which was provided by Intel Corporation. Differences in embryo locations between one frame and the next (distances of movements) were calculated as body movements per frame. This procedure was repeated for 5 min (9000 frames) and data strings of the coordinates of embryos were recorded.

Figure 2 shows an example of the recorded image and the results of template matching following use of the head area for the template image at $85 \mathrm{~h}$ of embryo incubation. Whole embryos and blood vessels are indicated by the yellow dotted lines and black arrows in Fig. 2A, respectively. The red rectangles indicate areas of the template image in which correct and incorrect matching was achieved. The embryo's head position was defined as the embryo's position, and was successfully detected (Fig. 2B). However, because template matching occasionally failed (Fig. 2C), incorrect detection data were removed using a digital low-pass filter (FIR filter, cut-off frequency of $2 \mathrm{~Hz}$ ), as described previously ${ }^{(14)}$. Calculation procedures for distances of body movements are summarized in Fig. 3. 
To investigate periodicity of body movements, power spectrum analyses were performed using discrete Fourier transform after applying a Hamming window ${ }^{(15,}$ 16). Analyses were performed over $5 \mathrm{~min}$ and the data were filtered with a cut-off frequency of $2 \mathrm{~Hz}$, corresponding with an effective frequency component from $3.3 \times 10-3$ to $2.0 \mathrm{~Hz}$.
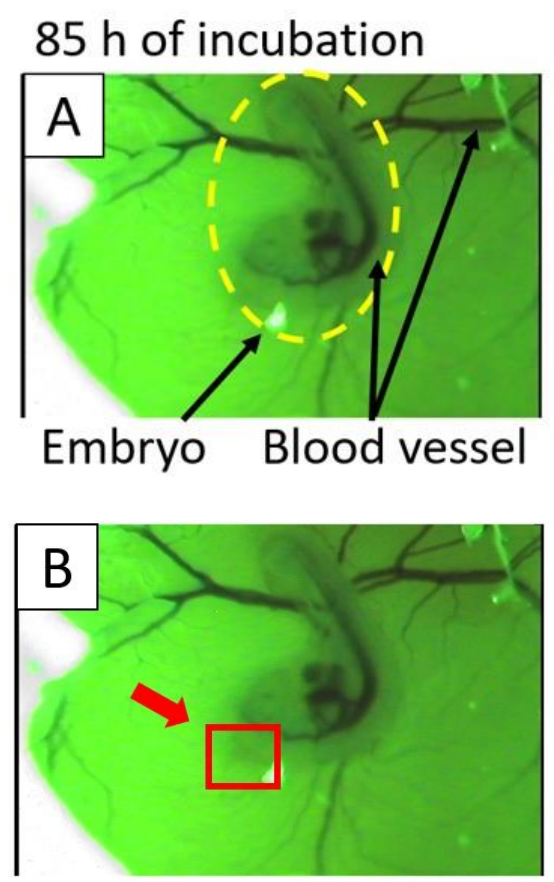

Correct Matching

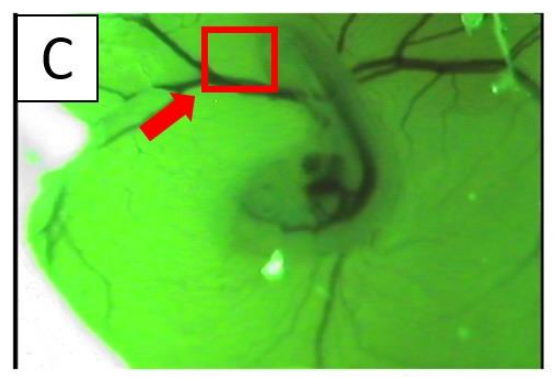

Incorrect Matching

Fig. 2. An example of recorded images and results of template matching following use of head areas as a template image after $85 \mathrm{~h}$ of embryo incubation; a whole embryo and its blood vessels are indicated by yellow dotted lines and black arrowheads in Fig 2A.

The position indicated by the red rectangle is the area matched to the template image. The embryo's head position (embryo position) was successfully detected in Fig 2B. An example of failed template matching is shown in Fig 2C.

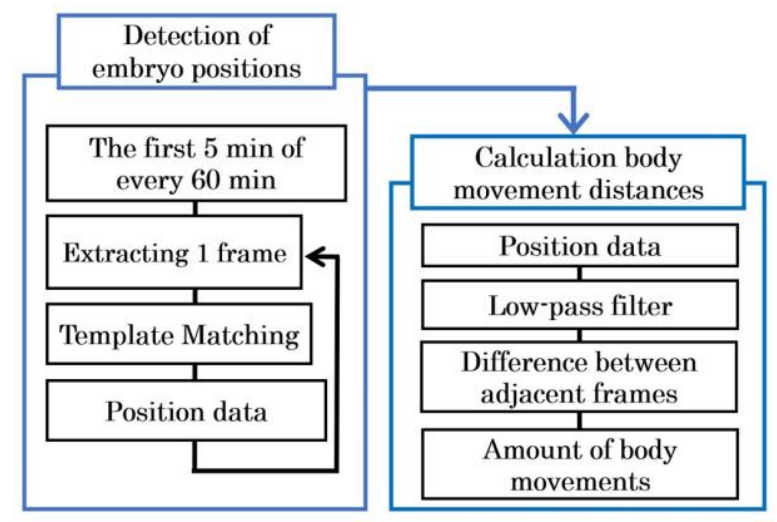

Fig. 3. A schematic diagram of calculation procedure for sizes of body movements.

\section{Results}

\subsection{Development of embryonic body movements}

Although images were recorded for eight embryos, only those for three individuals were suitable for continuous analyses of locations, angles, and measurement foci, and body movements of these three embryos were calculated using the abovementioned image processing method.

Figures 4, 5, and 6 show recorded embryos and indicate body movements over the first $5 \mathrm{~min}$ of each incubation time point. These data were used to characterize changes in body movement patterns. Images on the left side show chick embryos at each incubation time, and middle and right panels show relative distances (in pixels) of movements on X-and Y-axes and two-dimensional orbits of body movements during 5 -min periods at each incubation time point. The scales of vertical axes varied according to magnitudes of body movements. Because embryos were rotated to adjust the focus of the camera, the $\mathrm{X}-\mathrm{Y}$ direction is given as a relative coordinate, and hence, $\mathrm{X}$ and $\mathrm{Y}$ axes in the middle panels show directional characteristics of body movements.

As shown in Fig. 4, body movements of embryo A were not observed in $\mathrm{X}$ - or Y-axes at $72 \mathrm{~h}$ after the start of incubation, but these movements gradually began at $85 \mathrm{~h}$ after the start of incubation, albeit with limited sizes of movements. In addition, an in-phase periodicity was observed in movements on both $\mathrm{X}$ - and Y-axes. Frequencies of body movements did not change significantly at $100 \mathrm{~h}$ after the start of incubation, whereas cycles per body movement were approximately three times shorter. 
Furthermore, at $110 \mathrm{~h}$ after the start of incubation, sizes of body movements decreased and clear periodicity disappeared. However, at $125 \mathrm{~h}$ after the start of incubation, movements became energetic and complex, and based on $\mathrm{X}-\mathrm{Y}$ trajectories, these movements produced a horizontal circular pattern.

In embryo B (Fig. 5), no body movements were observed at $72 \mathrm{~h}$ after the start of incubation. Moreover, periodic movements began at $85 \mathrm{~h}$ after the start of incubation, as in Embryo A. However, frequencies of body movements in embryo B gradually increased thereafter and became complex with incubation time and embryonic development.

Body movements of embryo $\mathrm{C}$ had already started at $72 \mathrm{~h}$ after the start of incubation, and both periodicities and sizes of body movements were increased by $85 \mathrm{~h}$ after the start of incubation (Fig. 6). At this time, movements of embryo $\mathrm{C}$ showed significant periodicity and at around 100 $\mathrm{h}$ of incubation, body movements had no apparent directions and became complex and large. Although the
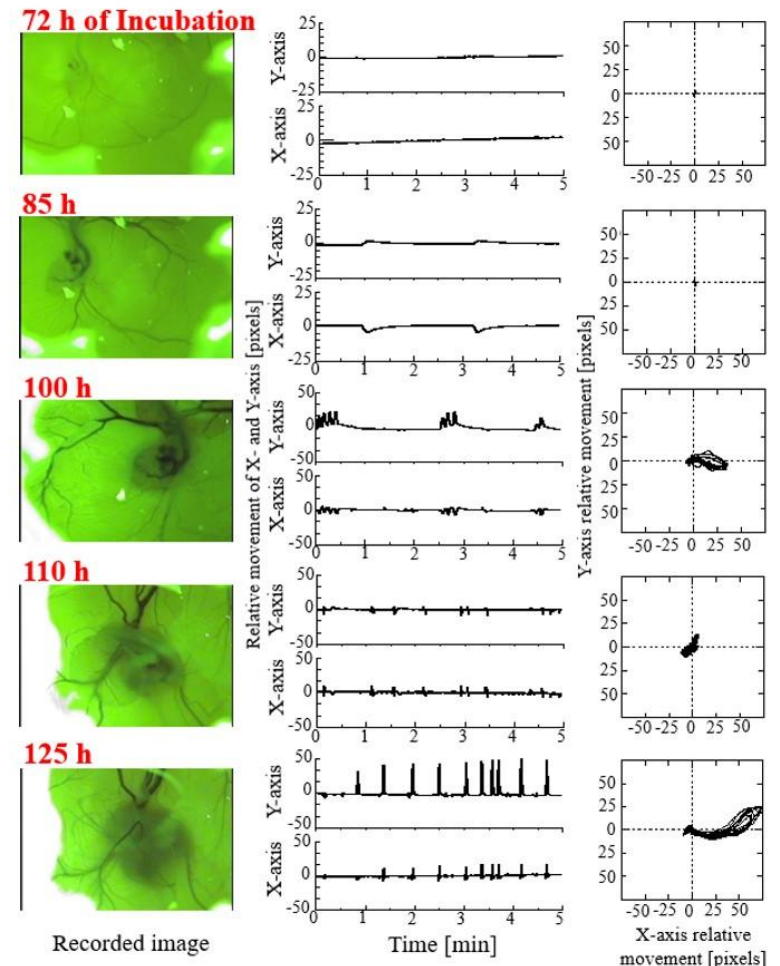

Fig. 4. Recorded images (left panels) and sizes of body movements (pixels) on X-and Y-axes (middle panel), and in the $\mathrm{X}-\mathrm{Y}$ (right panel) direction during 5-min time points at 72, 85, 100, 110, and $125 \mathrm{~h}$, respectively, in embryo A. Total relative body movements were determined and are expressed in pixels.
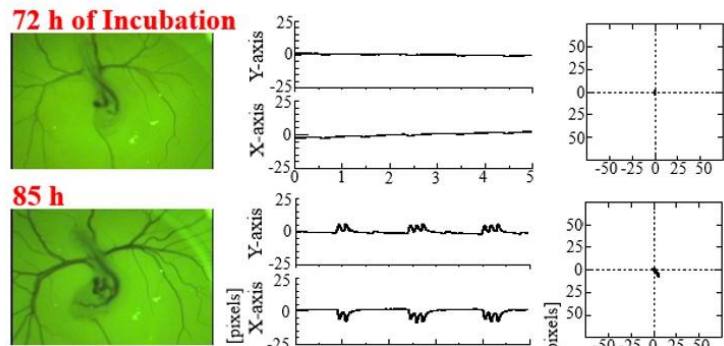

$100 \mathrm{~h}$

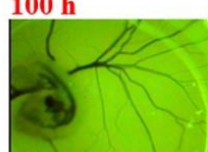

$110 \mathrm{~h}$

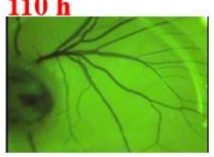

$125 \mathrm{~h}$

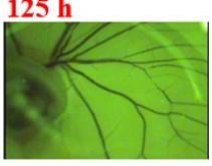

Recorded image
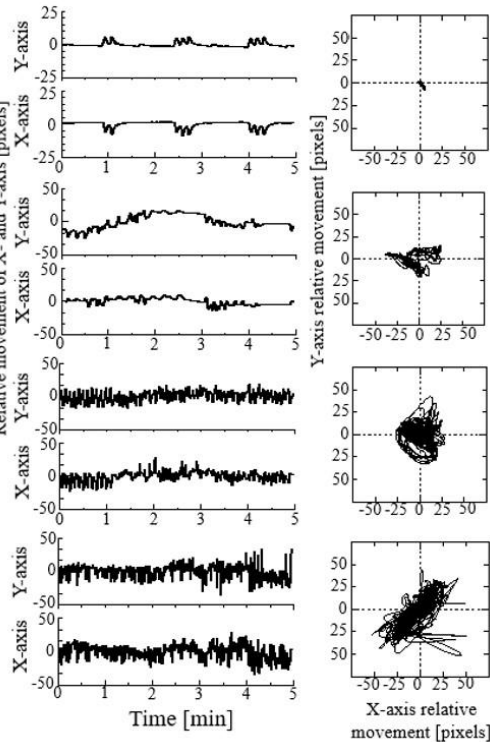

Fig. 5. Images of embryo B (left panels) and volumes of body movements on $\mathrm{X}$ - and Y-axes (middle panels), and in the $\mathrm{X}-\mathrm{Y}$ (right panels) direction.
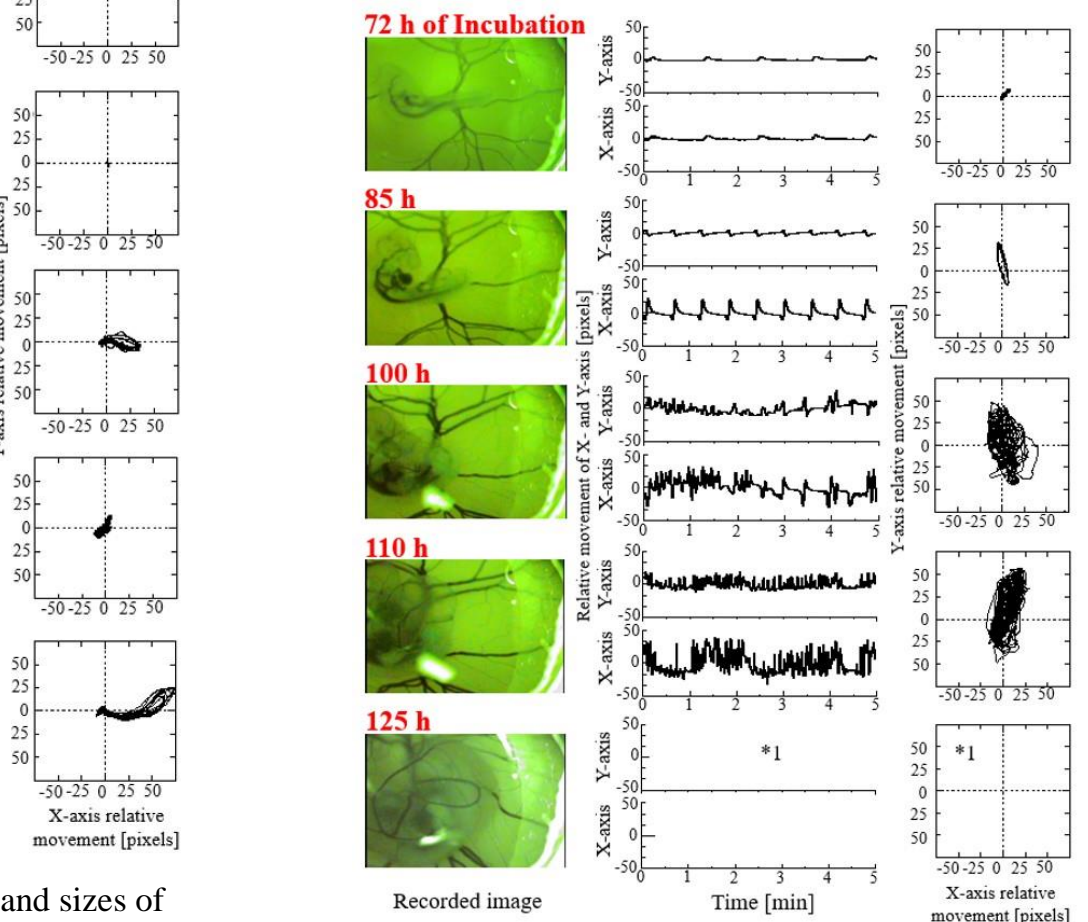

Fig. 6. The recorded images (left panels) and body movements of embryo B on X-and Y-axes (middle panels), and in the $\mathrm{X}-\mathrm{Y}$ (right panels) direction. Body movements at $125 \mathrm{~h}$ of incubation (*1) could not be measured because embryo B was obscured by a newly developed chorioallantoic membrane. 
frequencies of movements of embryo $\mathrm{C}$ were augmented at $125 \mathrm{~h}$ of incubation, determinations of the embryo positions using template matching failed thereafter because a chorioallantoic membrane grew between the CCD camera and the embryo. These observations indicate that the present template-matching method is suitable for embryos until around the 125th hour.

\subsection{Periodicity of body movements}

Although body movements of embryos $\mathrm{A}$ and $\mathrm{B}$ at around $72 \mathrm{~h}$ were transient and irregular, periodic movements were observed at around $85 \mathrm{~h}$ in all individuals, and these were pronounced on the Y-axis (Fig. 6) and subsequently became continuous and random. Thus, periodicity of body movements in these individuals was investigated using discrete Fourier transform (DFT) analyses (Fig. 7). At $85 \mathrm{~h}$ after the start of incubation, periodic peaks were approximately 148 (Embryo A), 99 (Embryo B), and $37 \mathrm{~s}$ (Embryo C) apart, respectively. Although the frequency of movements was shorter for embryo $\mathrm{C}$ than for the other individuals at the same incubation time, peaks of movements were approximately $74 \mathrm{~s}$ apart at $72 \mathrm{~h}$, similar to those of embryo B at $85 \mathrm{~h}$

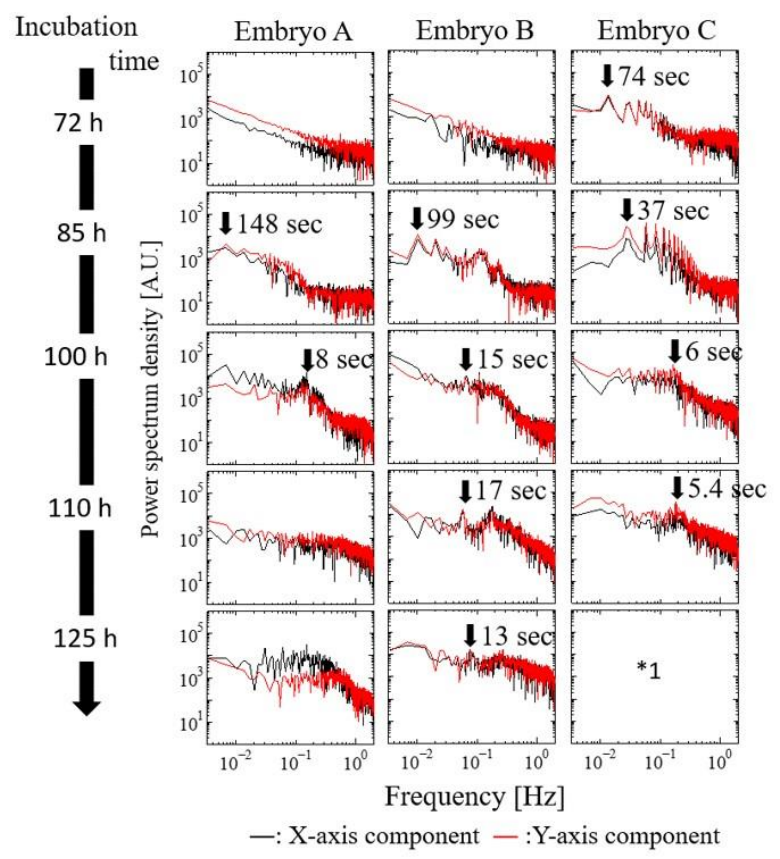

Fig. 7. Frequencies and components of body movements in $\mathrm{X}$ and $\mathrm{Y}$ directions at each incubation time; peak frequencies are indicated by arrows and cycle periods are indicated in $\mathrm{s}$ and are the inverse of frequencies in $\mathrm{Hz}$. Data for embryo C could not be calculated at the 125 th hour $(* 1)$ due to the lack of body movement data. after the start of incubation. All embryos had cyclical body movements at the 100th hour of incubation. However, these periods were shorter than those at $85 \mathrm{~h}$, and ranged from 148 to $8 \mathrm{~s}$ in embryo $\mathrm{A}$, from 99 to $15 \mathrm{~s}$ in embryo $\mathrm{B}$, and from 37 to $6 \mathrm{~s}$ in embryo $\mathrm{C}$, respectively. At the $110 \mathrm{~h}$ of incubation, movements followed a periodicity of approximately $17 \mathrm{~s}$ in embryo B and $5.4 \mathrm{~s}$ in embryo C, and had disappeared in embryo A. At the 125 th hour of incubation, periodicity of body movements remained only in embryo $\mathrm{B}$, although body movements of embryo $\mathrm{C}$ were not visible at this time point. These results (Fig. 7) indicate that cyclic periods between body movements gradually become shorter, and that periodicity is gradually replaced by irregular body movements.

\section{Discussions}

\subsection{Direct recording system and calculations of embryonic body movements}

Herein, we directly recorded embryonic development using a CCD camera, and calculated frequencies and periodicities of chick embryo body movements during the early stages of development from 72 to $125 \mathrm{~h}$ of incubation. The present direct recording system was developed and calculations were performed using template matching to determine distances of embryonic body movements. Although we tried to record body movements and calculate distances for eight individuals, data were only successfully generated for three embryos. Whereas embryo positions were detected incorrectly on occasion due to miss-template matching, these data were excluded using a low-pass filter during subsequent digital processing procedures (Fig. 2C). Among difficulties faced during these experiments, embryos are relatively transparent apart from the heart and blood vessels (left-images in Fig. 4-6). Thus, the edges of embryo heads that moved well were distinguished using a template from background images of the amnion. Moreover, to reduce failures of template matching, an image processing program was used to distinguish embryos and to emphasize their edges. In particular, we distinguished the head and/or eyes using a differential filter. Focus adjustments of the CCD camera were required to improve image sharpness.

This system for measuring embryonic body movements was effective until about $125 \mathrm{~h}$ (5 days) of incubation. In contrast, measurements of body movements 
were previously achieved using a laser or a record needle in contact with the egg shell, and this system detected body movements from around 7 days of incubation ${ }^{(17,18)}$. Thus, with the use of the present and previously described measurement systems, body movements can be investigated during all incubation periods.

\subsection{Body movement patterns in chick embryos at early stages of development}

Embryos commonly began moving with floating fluctuations after $72 \mathrm{~h}$ of incubation, and we assumed that this is common to all normally growing embryos. Subsequently, intermittent and irregular body movement patterns became cyclic and had circular orbits after $85 \mathrm{~h}$ of incubation, but then became irregular with increases in volumes of movement. Although the observed cyclic body movements had periods of 100-150 s at the $85 \mathrm{~h}$ incubation time point, these cyclic periods became shorter and then disappeared during further embryonic development until the 125 th hour. Although the causes of cyclic movements remain speculative in the absence of developed physiological functions, they may relate to the development of blood vessels, the lack of oxygen, or forces from the heart beat. Because these circular movements were observed in all of the present embryos, they may be necessary for normal growth and could be a significant milestone during embryonic development. However, further investigations are required to determine the presence of these cyclic body movements in abnormal embryos, and to confirm that they are common to all normally growing embryos.

Finally, the cyclic periods (approximately $74 \mathrm{~s}$ ) of body movements in embryo $\mathrm{C}$ at $72 \mathrm{~h}$ corresponded with those of embryo B at $85 \mathrm{~h}$ (approximately 99 s). Moreover, cyclic periods at other incubation time points were shorter for embryo $\mathrm{C}$ than for embryo $\mathrm{B}$, and the body size of embryo $\mathrm{C}$ at $72 \mathrm{~h}$ was similar to that of embryo B at $85 \mathrm{~h}$. These observations suggest that that embryo $\mathrm{C}$ developed earlier than embryo B, and that developmental patterns of body movement could be used to identify normal growth or delayed development.

Mortality rates of human fetuses are reportedly increased among embryos with limited body movements (13). Hence, the present system for continuous monitoring of body movements in chick embryos may offer a tool for diagnosing abnormal growth patterns. However, future studies are required to correlate distinctive body movement patterns and developmental patterns with embryonic growth in disease models and under conditions of privation, such as hypoxia.

\section{Acknowledgment}

A part of this study was supported by a Grant-in-Aid for Scientific Research ( K.M. JP19K04433) from the Japan Society for the Promotion of Science. The authors would like to thank Enago (www.enago.jp) for the English language review.

\section{Additional statement}

This research was approved by the Life Ethics Committee of NIT(KOSEN), Hakodate College.

\section{References}

(1) H. Tazawa, A. Ar, E. Gefen, K. Moriya, and J. T. Pearson: "Effects of incubator humidity on embryonic heart rate in the ostrich", Proc. 10th European Poultry Conference, pp.843-847, 1998

(2) H. Tazawa, K. Moriya, A. Tamura, T. Komoro, and R. Akiyama: "Ontogenetic study of thermoregulation in birds", Journal of Thermal Biology, Vol.26, pp.281-286, 2001

(3) A. Tamura, R. Akiyama, K. Moriya, E. M. Dzialowski, W. W. Burggren, and H. Tazawa: "Heart rate responses to cooling in emu hatchlings", Comparative Biochemistry and Physiology, Vol.134, Vol.4, pp.829-838, 2003

(4) R. Akiyama, A. Matsuhisa, J. T. Pearson, and H. Tazawa: "Long-term measurement of heart rate in chicken eggs", Comparative Biochemistry and Physiology, Part.A.124, pp.483-490, 1999

(5) K. Moriya, J. Höchel, J. T. Pearson, and H. Tazawa: "Cardiac rhythms in developing. chicks", Comparative Biochemistry and Physiology. Part.A.124, pp.463-470, 1999

(6) K. Moriya, J. T. Pearson, W. W. Burggren, A. AR, and H. Tazawa: "Continuous measurement of instantaneous heart rate and It's fluctuations before and after hatching in chickens", Journal of Experimental Biology, 203(Pt 5), pp.895-903, 2000

(7) K. Moriya, R. Akiyama, E. M. Dzialowski, W. W. Burggren, and H. Tazawa: "Development of heart rate circadian rhythm in chickens", Avian and Poultry Biology Reviews, Vol.15, No.3/4, pp.211-218, 2004. 
(8) K. Moriya, Y. Chiba and Y. Maruyama; "Effects of Acoustic Stimuli on the Heart Rate Fluctuations and Autonomic Nervous System Activity of Prenatal Chick Embryos", International Journal of Biomedical Soft Computing and Human Sciences, Vol .23 No.1, pp.19-25, 2018

(9) K. Moriya, Y. Chiba and Y. Maruyama; "Spectrum Analysis of Heart Rate Fluctuations in Hatched and Unhatched Prenatal Chick Embryos", Journal of the Institute of Industrial Applications Engineers, Vol.6 No.3, pp.107-111, 2018

(10) R. Akiyama, A. Kanbara, T. Komoro, N. Kataoka, H. Yoneta, K. Moriya, and H. Tazawa: "Continuous, long-term observation of early development of chicken embryos in ovo", New insights into fundamental physiology and peri-natal adaptation of domestic fowl, Special issue, pp.117-124. 2007

(11) H. Yoneta, R. Akiyama, W. Nakata, K. Moriya, and H. Tazawa: "Video analysis of body movements and their relation to the heart rate fluctuations in chick hatchlings", New insights into fundamental physiology and peri-natal adaptation of domestic fowl, Special issue, pp.57-68. 2007

(12) A. Yokota, K. Tenma, Y. Chiba, and K. Moriya, Growth and Body Movement Analysis Using Image Processing of Chick Embryos in Hypoxia Environment, Proceedings of the 7th IIAE International Conference on Industrial Application Engineering 2019, pp. 9-12, 2019

(13)E. Sadovsky, H. Yaffe : "Daily Fetal Movement Recording and Fetal Prognosis", Obstet Gynecol. , 41, pp.845-50, 1973

(14) K. Nakamura: "Design of FIR Filter with Smaller Number of Coefficients based on Compactly Supported Fluency Sampling Functions", Proceedings of 2003 IEEE Pacific Rim Conference on Communications, Computers and Signal Processing, pp.256-259, 2003

(15) Harris, F.J.: "On the use of windows for harmonic analysis with the discrete Fourier transform", Proceedings of the IEEE, Vol.66, Issue1, pp.51-83, 1978

(16) K. Moriya, I. Kurimoto, N. Ezaki, and M. Nakagawa: "Influences of Listening to Music in Study Break on Brain Activity and Parasympathetic Nervous System Activity", Journal of the Institute of Industrial Applications Engineers, Vol.6, No.1, pp.34-38, 2018

(17) Y. Suzuki, H. Musashi, and H. Tazawa: "Noninvasive heart rate monitoring system for avian embryos based on the ballistocardiogram", Med Biol Eng Comput. 27(4), pp.399-404, 1989

(18) H. Ono, R. Akiyama, Y. Sakamoto, J. T. Pearson, and H. Tazawa: "Ballistocardiogram of avian eggs determined by an electromagnetic induction coil", Med Biol Eng Comput, 35(4), pp.431-435, 1997 